\title{
Serum tumor necrosis factor-like weak inducer of apoptosis (sTWEAK) and IL-17 levels are associated with disease activity in systemic lupus erythematosus patients with and without nephritis
}

\author{
Mohammadreza Nakhjavani $^{1 \oplus}$, Sima Abediazar $^{2}{ }^{\circledR}$, Amir Ghorbanihaghjo $^{3^{*}}{ }^{\oplus}$, Niloofar Esmaeili $^{1}$, \\ Tala Pourlak ${ }^{2}$, Sepideh Zununi Vahed ${ }^{2}(\mathbb{D}$ \\ ${ }^{1}$ Department of Rheumatology, Connective Tissue Diseases Research Center, Tabriz University of Medical Sciences, Tabriz, Iran \\ ${ }^{2}$ Kidney Research Center, Tabriz University of Medical Sciences, Tabriz, Iran \\ ${ }^{3}$ Biotechnology Research Center, Tabriz University of Medical Sciences, Tabriz, Iran
}

\section{A R T I C L E I N F O}

\section{Article Type:}

Original

\section{Article History:}

Received: 5 January 2019

Accepted: 29 March 2019

Published online: 18 April 2019

\section{Keywords:}

Systemic lupus erythematosus, Lupus biomarkers, Nephritis, Chronicity index, Proteinuria, Activity index, End-stage renal disease, Dialysis, Renal replacement

\begin{abstract}
A B S T R A C T
Introduction: Lupus nephritis (LN) is one of the most severe signs of systemic lupus erythematosus (SLE) and rapid diagnosis of kidney damage remains an important concern for LN.

Objectives: The aim of this study was to investigate the association of the serum levels of tumor necrosis factor-like weak inducer of apoptosis (TWEAK) and interleukin 17 (IL-17) with SLE severity, renal involvement, and other clinical manifestations in lupus patients.

Patients and Methods: In order to determine a better biomarker for the detection of renal damage, this study evaluated the ability of serum TWEAK (sTWEAK) and IL-17 in lupus patients with $(n=25)$ and without $(n=25)$ nephritis and healthy controls $(n=39)$. Moreover, it compared the levels of these cytokines with disease activity and chronicity as well as traditional serum markers including complement $\mathrm{C} 3$ and $\mathrm{C} 4$, creatinine, and proteinuria in lupus patients.

Results: Increased levels of sTWEAK and IL-17 were observed in SLE and LN groups compared to healthy controls and non-LN groups, respectively. Significant positive associations were observed between serum TWEAK and IL-17 levels and systemic lupus erythematosus disease activity index (SLEDAI), proteinuria, nephritis activity index, and some clinical manifestations $(P<0.05)$. Discriminating the ability of the studied cytokines were not better than the utility of any markers individually.

Conclusion: The serum levels of TWEAK and IL-17 in the SLE and LN groups were significantly higher than the control group and both markers were indicative of the renal disease severity; therefore, they could possibly indicate renal involvement in the lupus patients.
\end{abstract}

Implication for health policy/practice/research/medical education:

A reliable marker that can reveal the renal disease activity in lupus patients is desirable. In the present study, we evaluated the diagnostic values of serum tumor necrosis factor-like weak inducer of apoptosis (TWEAK) and IL-17 in serum samples of lupus patients with and without nephritis and healthy controls.

Please cite this paper as: Nakhjavani MR, Abediazar S, Ghorbanihaghjo A, Esmaeili N, Pourlak T, Zununi Vahed S. Serum tumor necrosis factor-like weak inducer of apoptosis (sTWEAK) and IL-17 levels are associated with disease activity in systemic lupus erythematosus patients with and without nephritis. J Renal Inj Prev. 2019;8(3):204-210. DOI: 10.15171/jrip.2019.38.

\section{Introduction}

Lupus nephritis (LN) is one of the most severe signs of systemic lupus erythematosus (SLE), a complex inflammatory autoimmune disease, which leads to morbidity and mortality of patients. In spite of improvement in the treatment and survival rates of LN patients, its prognosis remains inacceptable. The prognosis of LN demands developing novel approaches with high sensitivity and specificity for the onset or relapse of kidney disease activity; consequently, permitting initiation of management plans at an earlier and proper time (1). Late LN diagnosis associates with a higher incidence of kidney deficiency and development of end-stage renal disease (ESRD), emphasizing the significance of early diagnosis. For these reasons, the identification of novel biomarkers is clearly needed.

Tumor necrosis factor-like weak inducer of apoptosis (TWEAK), is a member of TNF-ligand superfamily (2, 
3) and a multifunctional cytokine in kidney injury which is produced by monocytes and macrophages. TWEAK is involved in tissue repair and regeneration (4). TNFlike weak inducer of apoptosis by persistent activation of the NF- $\kappa \mathrm{B}$ pathway, can contribute to autoimmune diseases (5). Moreover, TWEAK up-regulates other chemokines, cytokines, and pro-inflammatory mediators that are involved in LN (6). Furthermore, it increases the initiation of apoptosis (2) and activates TGF- $\beta$ in renal proximal tubule cells (7). The biological activity of TWEAK is mediated through its receptor, fibroblast growth factor-inducible 14 (Fn14) (8). The TWEAKFn14 pathway activates the NF- $\kappa B$ signaling pathway to result in inflammation, cell proliferation, angiogenesis, and apoptosis (9). Moreover, TWEAK-Fn14 signaling is associated with the pathogenesis of renal damage and LN $(8,10)$ since it is involved in numerous processes that trigger LN such as inflammation, mesangial cell proliferation, vascular activation, kidney cell death, and fibrosis $(8,11)$. Based on animal models, TWEAK has also a regulatory role in type I IFN pathway in LN (10). It is essential to note that TWEAK regulates different urinary cytokines and chemokines (MCP-1, IL-6, and IP-10) that are associated with the disease activity. Being involved in the inflammatory cascade, TWEAK is an attractive mediator that can be considered as a potential biomarker and a promising predictor of flare in LN.

Interleukin-17 (IL-17), a pro-inflammatory cytokine, is produced by $\mathrm{T}$ cells ( $\mathrm{T}$ helper, $\gamma \delta \mathrm{T}$, and Th17 cells) (12, 13). The number of $\mathrm{CD}^{+}$effector T cells that secret IL-17 are higher in SLE patients and its plasma level is associated with disease activity in SLE patients without nephritis (14, 15). In LN, the IL-17 level, is higher in active diseases in comparison to remission and it is associated with level of anti-dsDNA and proteinuria (16). Additionally, in glomeruli, the expression of IL-17 is increased in diffuse proliferative diseases and correlated with the histological activity index in comparison to healthy kidneys (17).

\section{Objectives}

The results of recent studies indicate the important role of IL-17 and TWEAK in the pathogenesis of inflammatory and autoimmune diseases, such as rheumatoid arthritis and lupus. Nevertheless, no study has so far been conducted to examine these two factors simultaneously as well as their correlation with each other and with the illness severity in the LN patient. Therefore, the aim of this study was to investigate the association of the serum levels of TWEAK and IL-17 with SLE severity, renal involvement, and other clinical manifestations in lupus patients.

\section{Patients and Methods}

\section{Study population}

The cross-sectional study included 50 SLE patients from the Imam-Reza hospital, Tabriz, Iran, consisting of $25 \mathrm{LN}$ patients with active renal disease at the time of the visit and 25 non-LN SLE patients. LN cases were defined as SLE patients with renal involvement based on clinical manifestation and renal biopsies. Non-LN SLE cases were defined as SLE patients without any signs of recent and previous renal involvement. Moreover, 39 healthy individuals with no known history of kidney or autoimmune disease were recruited. An informed consent was obtained from all patients enrolled in the present study. Cases were collected within one year and met the SLE diagnostic criteria according to the American College of Rheumatology. Cases with active infection at the time of sampling, overlap syndrome, any other glomerulopathy, diabetes mellitus, history of malignancies, urinary tract infection, and those with ESRD under dialysis or renal replacement therapy were excluded.

\section{Clinical and laboratory measurements}

On the day of the sampling, the information about patient's demographic characteristics, medications, and disease activity was recorded. For each patient, fresh blood sample $(4 \mathrm{~mL})$ was collected. Sera were frozen after collection (within 2 hours) and stored at $-80^{\circ} \mathrm{C}$ until further analysis. Serum creatinine and BUN levels, some biochemical parameters, complement (C3 and C4), antinuclear antibodies (ANA), anti-dsDNA antibody (antidsDNA), and the amount of proteinuria in 24-hour urine samples were assessed. Serum levels of TWEAK and IL-17 were determined by the enzyme-linked immunosorbent assay (ELISA) based on the manufacturer's instructions (ZellBio GmbH, Cat No: ZB-138223c-H9648, Germany). Plates were read at $450 \mathrm{~nm}$. All measurements were made in triplicate.

\section{Ethical approval}

The study was approved by the Clinical Research Ethics Committee of the Tabriz University of Medical Sciences, Tabriz, Iran (Ethical code: IR.TBZMED.REC.1396.599) and has therefore been performed in accordance with the ethical standards laid down in the 1964 Declaration of Helsinki and its later amendments. The protocol of the study was clarified to all participants and written informed consent was achieved from the patients. The study was extracted from a residency thesis of Dr. Niloofar Esmaeili at Tabriz University of Medical Sciences, Tabriz, Iran (Research code; 58856).

\section{Statistical analysis}

Data were given as mean \pm SD or median with interquartile range (IQR) for normally and non-normally distributed variables, respectively. Since the data were not normally distributed, the data therefore were expressed as the median and IQR and the differences between the groups were analyzed by the Mann-Whitney $U$ and Kruskal-Wallis tests. Comparisons of categorical variables 
were conducted using Chi-square testing. Correlations were performed using the Spearman's rank correlation coefficient to test correlations between the variables. Statistical analysis was performed using SPSS statistical software, version 16.0 (SPSS, Chicago, IL). $P<0.05$ was considered significant.

\section{Results}

\section{Patient characteristics}

The demographic characteristics of the studied groups are presented in Table 1. All 50 SLE patients with a mean of $36.7 \pm 11$ years $($ male/female $=10 / 40)$ were included in the present study. Moreover, the control group included 39 age/gender matched healthy subjects with a mean age of $38.4 \pm 9.7$ years old. In all groups, more than $80 \%$ of the individuals were women. The LN group had a mean age of $35.4 \pm 11.7$ years old. Most of the studied SLE cases $(88 \%)$ had an active disease (SLEDAI scores $\geq 6$ ). Urine proteinuria ( 24 hours) $>500 \mathrm{mg} / \mathrm{d}$ was defined as positive for proteinuria. Significantly, higher proteinuria [Median (IGR) of $1368 \mathrm{mg} / \mathrm{d}$ (926 to $2000 \mathrm{mg} / \mathrm{d}$ ) versus $125 \mathrm{mg} / \mathrm{d}(92-170 \mathrm{mg} / \mathrm{d})$ ], and SLEDAI scores [Median (IGR) of 10 (8-14) versus 6 (5.5-9)] and lower levels of the complement C3 [Median (IGR) of $22.32 \pm 10.98 \mathrm{mg} /$ $\mathrm{dL}$ versus $72.28 \pm 24.0 \mathrm{mg} / \mathrm{dL}$ ] were observed in $\mathrm{LN}$ group compared to non-LN patients, $P \leq 0.001$ (Table 1).

\section{Systemic organ involvements}

Skin disease (52\%), articular involvement (52\%), and renal disease $(50 \%)$ were the most common manifestations of the SLE patients. Twenty-four percent of SLE patients had leucopenia, $16 \%$ had a prevalence of serositis, $12 \%$ had thrombocytopenia, and $10 \%$ had Central nervous system (CNS) involvement. The renal involvement of SLE patients was diagnosed at the time of sampling and confirmed histologically. Patients with LN had a higher prevalence of serositis (20\% versus $12 \%, P=0.700)$, hematuria $(40 \%$ versus $0 \%, P=0.001)$, and CNS involvement ( $16 \%$ versus $4 \%, P=0.350$ ) than non-LN patients (Table 1 ).
Serum levels of TWEAK and IL-17 in different groups As shown in Figure 1A, a higher serum level of TWEAK was observed in the SLE group (with or without LN) as compared to controls [Median $(\mathrm{IQR})=69.0$ (64.8-78.3) versus $65.2(53.3-70.5), P<0001]$. Its level was even significantly higher in LN group in comparison to nonLN group [Median $(\mathrm{IQR})=76.40(67.9-83.50)$ versus 66.7 (63.3-69.9), $P=0.002$ ] (Figure 1B). Moreover, the levels of serum IL-17 were increased in all SLE cases [Median $(\mathrm{IQR})=23.8(21.0-27.3)$ versus $21.4(20.5-24.5), P=$ 0.004 ] (Figure $1 \mathrm{C}$ ) and patients with LN [Median (IQR) $=25.2(21.22-30.6), P=0.077$ ] (Figure 1D) as compared to controls and those without $\mathrm{LN}$, respectively.

Based on organ involvement, levels of sTWEAK and IL17 in the SLE patients are presented in Table 2. Medians of sTWAEK increased significantly in SLE patients with neurological $(P=0.001)$ and skin $(P=0.008)$ involvement. Likewise, levels of IL-17 were significantly higher in patients with CNS involvement $(P=0.001)$ and serositis $(P=0.004)$ when compared to those with normal organs. Increased levels of the mentioned cytokines were observed in patients with other clinical manifestations including arteritis, thrombocytopenia, serositis, and leucopenia, however, they were not statistically significant when compared to normal organs $(P>0.05)$.

\section{Correlations of TWEAK and IL-17 and traditional parameters}

There was a significant internal correlation between serum levels of TWEAK with IL-17 in all SLE patients $(\mathrm{r}=471, P$ $=0.018)$ and in LN patients $(\mathrm{r}=0.471, P=0.018)$. In LN group, there was a significant positive association between serum TWEAK and IL-17 levels with SLEDAI ( $r=0.513, P$ $=0.009$ and $\mathrm{r}=0.883, P<0.001$ respectively) and with level of proteinuria $(\mathrm{r}=0.668, P<0.001$ and $\mathrm{r}=0.461, P=0.020$ respectively) (Table 3 ). More importantly, both sTWEAK $(\mathrm{r}=0.491, P=0.013)$ and IL-17 $(\mathrm{r}=0.463, P=0.020)$ markers associated with nephritis activity index, while only sTWEAK correlated with chronicity index $(r=0.482$,

Table 1. Demographic and baseline clinical data

\begin{tabular}{|c|c|c|c|}
\hline Characteristics/Groups & Non-LN group $(n=25)$ & LN group $(n=25)$ & $P$ value $^{\mathrm{a}}$ \\
\hline Female (No., \%) & $20(80)$ & $20(80)$ & 1 \\
\hline Age, mean \pm SD $(y)$ & $38 \pm 10.9$ & $35.4 \pm 11.7$ & 0.429 \\
\hline C3 (mg/dL) & $72.28 \pm 24.0$ & $22.32 \pm 10.98$ & $<0.001$ \\
\hline $\mathrm{C} 4(\mathrm{mg} / \mathrm{dL})^{\mathrm{b}}$ & $9(8$ to 10$)$ & 6 to 10$) 8($ & 0.136 \\
\hline Anti-dsDNA ${ }^{b}$ & 9 (8 to 11$)$ & 16 (12 to 43$)$ & 0.002 \\
\hline Creatinine $(\mathrm{mg} / \mathrm{dL})$ & $0.87 \pm 0.10$ & $1.14 \pm 0.22$ & $<0.001$ \\
\hline Proteinuria $(\mathrm{mg} / 24 \mathrm{~h})^{\mathrm{b}}$ & 125 (92 to 170 ) & 1368 (926 to 2000) & $<0.001$ \\
\hline SLEDAI ${ }^{b}$ & 6 (5.5 to 9) & 10 (8 to 14$)$ & 0.001 \\
\hline Activity index $\pm S D$ & - & $10.8 \pm 3.0$ & - \\
\hline Chronicity index ${ }^{b}$ & - & 2 (2 to 4$)$ & - \\
\hline
\end{tabular}

Abbreviations: Anti-dsDNA, anti-double strand DNA; SLE, systemic lupus erythematosus; SLEDAl, systemic lupus erythematosus disease activity index; $\mathrm{C} 3 / \mathrm{C} 4$, complement components.

The quantity data are expressed as mean \pm SD.

${ }^{\mathrm{a}}$ Lupus nephritis versus non-lupus nephritis; ${ }^{\mathrm{b}}$ Median, Interquartile Range (IRQ). 

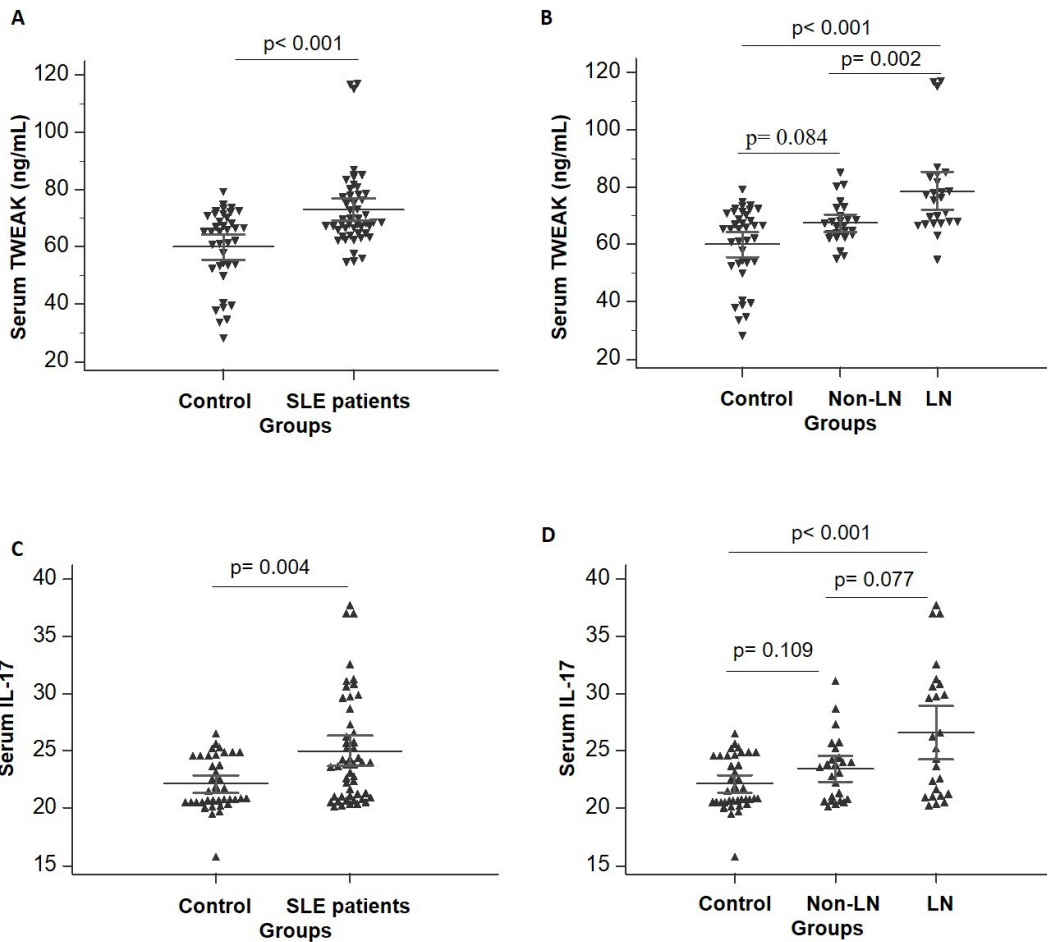

Figure 1. Serum level of TWEAK and IL-17 in the studied groups. (A,B) Serum levels of TWEAK between healthy and SLE groups with and without nephritis and $(C, D)$ Serum levels of IL-17 between healthy and SLE groups with and without nephritis. Values are the mean \pm standard deviation. SLE; systemic lupus erythematosus.

$0.015)$ and serum creatinine levels $(r=0.483, P=0.014)$ in LN group. Additionally, statistically significant positive correlations exited between IL-17 and SLEDAI $(r=0.675$, $P<0.001)$, C4 $(\mathrm{r}=0.404, P=0.045)$, and ANA $(\mathrm{r}=0.461, P$ $=0.02)$ in non-LN patients. Similarly, serum TWEAK was correlated with SLEDI $(\mathrm{r}=0.666, P<0.001)$ and serum ANA levels $(\mathrm{r}=0.444, P=0.026)$ in non-LN SLE patients. Correlations between serum levels of TWEAK and IL-17 and clinical manifestations of the SLE cases are listed in Table 3.

\section{Receiver operating characteristic curves analysis}

Receiver operating characteristic (ROC) curves for serum TWEAK and IL-17 were calculated for distinguishing SLE patients from healthy controls and also SLE patients with LN from those with non-renal involvement. The diagnostic performance of IL-17 and sTWEAK presented lower sensitivity and specificity (Table 4). Receiver operating characteristic analysis indicated that serum levels of TWEAK (area under the curve $($ AUC $)=0.728$ ) with $90 \%$ sensitivity and $46.15 \%$ specificity and IL-17 (AUC $=0.681$ ) with $34 \%$ sensitivity and $97.44 \%$ specificity could discriminate SLE patients from healthy controls. However, each parameter alone or their combination failed to differentiate most of LN patients from SLE patients with both high sensitivity and specificity (Table 4).

\section{Discussion}

Assessing renal involvement has become an important part of LN patients' evaluation. In the present study, we revealed that high levels of serum TWEAK and IL-17 were correlated with SLEDAI, serum creatinine, abnormal

Table 2. Levels of serum TWEAK and IL-17 based on organ involvement in the SLE patients

\begin{tabular}{|c|c|c|c|c|c|c|}
\hline \multirow{2}{*}{$\begin{array}{l}\text { Serum factors } \\
\text { Organ (N) }\end{array}$} & \multicolumn{3}{|l|}{ TWEAK } & \multicolumn{3}{|l|}{ IL-17 } \\
\hline & Not-involved & Involved & P value $^{a}$ & Not-involved & Involved & P value $^{a}$ \\
\hline CNS (45/5) & $68.0(64.5-76.4)$ & $115.9(89.9-116.8)$ & 0.001 & $23.6(20.9-25.6)$ & $32.6(31.1-90.6)$ & 0.001 \\
\hline Rash (24/26) & $75.8(67.2-84.0)$ & 67.7 (62.9-69.9) & 0.008 & $24.9(21.2-30.2)$ & $23.7(20.7-25.2)$ & 0.151 \\
\hline Arthritis $(24 / 26)$ & $68.2(66.7-75.8)$ & $71.3(64.1-80.7)$ & 0.503 & $23.2(20.9-25.4)$ & $23.9(21.2-29.7)$ & 0.327 \\
\hline Leucopenia (38/12) & $68.2(64.5-80.1)$ & $69.7(65.7-75.6)$ & 0.982 & $23.8(21.0-27.3)$ & $22.3(20.8-28.0)$ & 0.725 \\
\hline Serositis (42/8) & $69.0(64.5-77.2)$ & 73.1 (66.6-99.2) & 0.278 & $23.3(20.9-25.2)$ & $29.2(26.0-35.1)$ & 0.004 \\
\hline Thrombocytopenia (44/6) & $69.0(64.3-78.5)$ & $69.0(66.7-73.0)$ & 0.834 & 23.7 (20.9-26.9) & $24.9(22.1-30.6)$ & 0.438 \\
\hline
\end{tabular}

CNS: Central nervous system, SLE: systemic lupus erythematosus.

a Median, Interquartile Range (IRQ), Mann-Whitney test. $P<0.05$ was considered significant. 
Table 3. Correlations of serum TWEAK and IL-17 with demographic, clinical, and laboratory data of SLE patients with nephritis

\begin{tabular}{|c|c|c|c|c|}
\hline \multirow[b]{2}{*}{ Variable } & \multicolumn{2}{|c|}{ Serum TWEAK } & \multicolumn{2}{|c|}{ Serum IL-17 } \\
\hline & $\begin{array}{c}\text { Spearman's } \\
\text { rho }\end{array}$ & $P$ value & $\begin{array}{c}\text { Spearman's } \\
\text { rho }\end{array}$ & $P$ value \\
\hline Age & 0.447 & 0.025 & 0.176 & 0.399 \\
\hline Gender & 0.0 & 1 & 0.055 & 0.792 \\
\hline SLEDAI & 0.513 & 0.009 & 0.883 & $<0.001$ \\
\hline Creatinine & 0.483 & 0.014 & 0.204 & 0.327 \\
\hline Proteinuria & 0.668 & $<0.001$ & 0.461 & 0.020 \\
\hline C3 & -0.202 & 0.333 & -0.157 & 0.454 \\
\hline C4 & 0.026 & 0.901 & 0.064 & 0.762 \\
\hline ANA & 0.113 & 0.590 & 0.0 & 1 \\
\hline anti-dsDNA & 0.289 & 0.161 & 0.370 & 0.065 \\
\hline Activity index & 0.491 & 0.013 & 0.463 & 0.020 \\
\hline Chronicity index & 0.482 & 0.015 & 0.040 & 0.848 \\
\hline Hematuria & 0.260 & 0.209 & 0.215 & 0.302 \\
\hline CNS & 0.635 & 0.001 & 0.575 & 0.003 \\
\hline Rash & -0.286 & 0.166 & -0.013 & 0.951 \\
\hline Arteritis & -0.294 & 0.153 & 0.260 & 0.209 \\
\hline Leucopenia & -0.026 & 0.902 & 0.091 & 0.666 \\
\hline Serositis & 0.333 & 0.104 & 0.541 & 0.005 \\
\hline Thrombocytopenia & 0.123 & 0.559 & 0.102 & 0.627 \\
\hline
\end{tabular}

Anti-dsDNA: Anti-double-stranded DNA; SLEDAI: systemic lupus erythematosus disease activity index, C3/C4: complement components. $P<0.05$ was considered significant.

24-hour urine proteinuria, and LN activity index. Our results suggested that serum TWEAK and IL-17 increased parallel to the severity of kidney damage, confirming the earlier discovery of the association of these markers with renal damage.

TWEAK can stimulate macrophage migration through the down-regulation of IL-10 expression and its level is elevated in the renal tissue of SLE patients with nephritis, and in the urine of cases with active LN (18). Elevated levels of urinary TWEAK are also evident in LN patients $(2,19)$. Similar study reported that the urinary TWEAK (uTWEAK) was significantly higher in the LN patients than in the lupus and control groups and found a significant correlation with disease severity (20). In addition, high uTWEAK levels could predict nephritis in SLE patients with a high odds ratio of 7.36 (CI: 2.2524.07) (2). Thus, its diagnostic value was more than antidsDNA antibodies and it can be considered as a diseasemonitoring biomarker (19). Furthermore, uTWEAK was correlated with the renal SLEDAI score as well as other traditional biomarkers of LN activity such as antidsDNA antibodies and complement $(2,5)$. Hence, the urinary TWEAK level may be a novel biomarker of LN activity status $(9,18)$. These findings all suggest that the TWEAK can be an important marker for the diagnosis of the severity of lupus and LN. However, the LN cases could be better detected using UTWEAK (21). The result of the present study was in line with these reports, however, it was in contrast with the result of the study by Schwartz et al. They showed that sTWEAK level did not correlate with the presence of LN or the degree of nephritis activity (19). Nevertheless, we found a positive significant correlation between sTWEAK and nephritis activity and chronicity indices.

In this study, the IL-17 level in the lupus patients was higher than that in the control group, but no significant difference was observed between LN patients, non-LN, and healthy groups. Similar studies have reported significant correlations between the IL-17 level and disease severity in patients with lupus (22) and LN (16). In patients with LN, IL-17 was significantly higher in active disease compared with remission state and was correlated with dsDNA and proteinuria (16). Additionally, two other studies reported that the IL-17 levels were associated with the SLE disease activity $(22,23)$. Another study underlined that the IL-17 is a potent cytokine with severe pre-inflammatory activity that is abnormally produced in lupus patients (24). It was stated that the IL-17 level in the active LN patients was higher in the controls and treated lupus patients, and was elevated with an exacerbation in the lupus disease severity. It was also observed that, the high level of IL-17 is a predictor of inappropriate histopathologic response of LN (20). Our results are consistent with these studies. However, Raymond et al showed that the level of IL-17 in lupus patients did not differ significantly from those in the control group and did not show a significant correlation

Table 4. Performance in evaluating renal involvement

\begin{tabular}{|c|c|c|c|c|c|c|c|c|}
\hline & AUC & $P$ value & Sensitivity & Specificity & Youden & Associatedcriterion & LR+ & LR- \\
\hline \multicolumn{9}{|c|}{ SLE from controls } \\
\hline TWEAK & 0.728 & $<0.001$ & 90 [78.2-96.7] & 46.15 [30.1-62.8] & 0.36 & $>62.1$ & 1.67 & 0.22 \\
\hline IL-17 & 0.681 [0.57 to 0.79$]$ & 0.0013 & $34[21.2-48.8]$ & 97.44 [86.5-99.9] & 0.314 & $>25.63$ & 13.26 & 0.68 \\
\hline Combined & 0.763 [0.66 to 0.86$]$ & $<0.001$ & & & & & & \\
\hline \multicolumn{9}{|c|}{ LN from non-LN } \\
\hline TWEAK & 0.752 [0.610 to 0.863 ] & 0.0003 & $56[34.9-75.6]$ & 88 [68.8-97.5] & 0.44 & $>75$ & 4.67 & 0.50 \\
\hline IL-17 & 0.646 [0.498 to 0.776$]$ & 0.0725 & $48[27.8-68.7]$ & $88[68.8-97.5]$ & 0.36 & $>25.8$ & 4 & 0.59 \\
\hline Combined & 0.763 [0.629 to 0.897$]$ & 0.001 & & & & & & \\
\hline
\end{tabular}

AUC: Area under the Curve. $P<0.05$ was considered significant. 
with the illness severity (22). In the analysis of the above studies, it can be seen that IL-17 plays an important role in the pathophysiology of lupus and LN. While it was observed in this research and some other studies, that increased IL-17 levels may even be associated with exacerbation of the disease. Hence, drugs likely to inhibit this cytokine may be effective in controlling the disease.

Elevated levels of sTWEAK and IL-17 in our LN group was associated with clinical manifestations including arteritis, thrombocytopenia, serositis, and leucopenia. However, differences in serum levels of these factors according to the various organ involvement were not statistically significant. It has been stated that the TWEAK levels in serum and CSF were greater in patients with lupus than in people without autoimmune disease, while its increase in CSF can cause lupus neurological manifestations (25). Likewise, in the present study, the elevated levels of sTWEAK and IL-17 were correlated with CNS involvement in the LN group. All of these data confirm that levels of TWEAK and IL-17 having biologically reasonable functions in the pathology of LN, hence, it can be intriguing biomarkers for LN. However, ROC analysis in the present study indicated that serum TWEAK and IL-17 did not have high accuracy, sensitivity and specify to discriminate all/most of LN patients from non-LN patients.

\section{Conclusion}

In conclusion, the results of our study exhibited that the TWEAK and IL-17 levels were significantly higher in the lupus patients, while, these two cytokines showed a significant and direct correlation with the severity of lupus and renal involvement. Although our study revealed higher serum levels of TWEAK and IL-17 in SLE patients with organ involvement, some of them were not significant.

\section{Limitations of the study}

We believe that relatively small sample size is a limitation of our study. The strength of the present study was that we had a control group and compared the serum marker levels between SLE patients with and without LN and normal subjects. Moreover, the combined effect of the studied cytokines was evaluated in the diagnosis of nephritis in SLE patients.

\section{Authors' contribution}

All authors contributed to the study. MRN and SA designed the study and selected the cases. NS did sampling. AGH and TP performed experimental analysis and interpretation of the data. SZV prepared the draft. All authors read and approved the final manuscript.

\section{Conflicts interests}

The authors declared no potential conflicts of interest with respect to the research, authorship, and/or publication of this article.

\section{Ethical considerations}

Ethical issues (including plagiarism, data fabrication, double publication) have been completely observed by the authors.

\section{Funding/Support}

This research was funded by the Connective Tissue Diseases Research Center of Tabriz University of Medical Sciences, Tabriz, Iran (Grant \# 5/D/577304).

\section{References}

1. Saxena R, Mahajan T, Mohan C. Lupus nephritis: current update. Arthritis Res Ther. 2011;13:240. doi: 10.1186/ ar3378.

2. Schwartz N, Su L, Burkly LC, Mackay M, Aranow C, Kollaros M, et al. Urinary TWEAK and the activity of lupus nephritis. J Autoimmun. 2006;27:242-50. doi: 10.1016/j. jaut.2006.12.003.

3. Soliman S, Mohan C. Lupus nephritis biomarkers. Clin Immunol. 2017;185:10-20. doi: 10.1016/j.clim.2016.08.001.

4. Wiley SR, Winkles JA. TWEAK, a member of the TNF superfamily, is a multifunctional cytokine that binds the TweakR/Fn14 receptor. Cytokine Growth Factor Rev. 2003;14:241-9.

5. Adhya Z, Borozdenkova S, Karim MY. The role of cytokines as biomarkers in systemic lupus erythematosus and lupus nephritis. Nephrol Dial Transplant. 2011;26:3273-80. doi: 10.1093/ndt/gfq860.

6. Chicheportiche Y, Chicheportiche R, Sizing I, Thompson J, Benjamin CB, Ambrose C, et al. Proinflammatory activity of TWEAK on human dermal fibroblasts and synoviocytes: blocking and enhancing effects of anti-TWEAK monoclonal antibodies. Arthritis Res. 2002;4:126-33. doi: 10.1186/ar388.

7. Liu Z, Xue L, Liu Z, Huang J, Wen J, Hu J, et al. Tumor Necrosis Factor-Like Weak Inducer of Apoptosis Accelerates the Progression of Renal Fibrosis in Lupus Nephritis by Activating SMAD and p38 MAPK in TGF-betal Signaling Pathway. Mediators Inflamm. 2016;2016:8986451. doi: 10.1155/2016/8986451.

8. Michaelson JS, Wisniacki N, Burkly LC, Putterman C. Role of TWEAK in lupus nephritis: a bench-to-bedside review. J Autoimmun. 2012;39:130-42. doi: 10.1016/j. jaut.2012.05.003.

9. Armstrong CL, Galisteo R, Brown SA, Winkles JA. TWEAK activation of the non-canonical NF-kappaB signaling pathway differentially regulates melanoma and prostate cancer cell invasion. Oncotarget. 2016;7:81474-92. doi: 10.18632/oncotarget.13034.

10. Xue L, Liu L, Huang J, Wen J, Yang R, Bo L, et al. Tumor Necrosis Factor-Like Weak Inducer of Apoptosis Activates Type I Interferon Signals in Lupus Nephritis. Biomed Res Int. 2017;2017:4927376. doi: 10.1155/2017/4927376.

11. Chen J, Wei L, Xia Y. Roles of tumour necrosis factorrelated weak inducer of apoptosis/fibroblast growth factorinducible 14 pathway in lupus nephritis. Nephrology 
(Carlton). 2017;22:101-6. doi: 10.1111/nep.12957.

12. Jin W, Dong C. IL-17 cytokines in immunity and inflammation. Emerg Microbes Infect. 2013;2:e60. doi: 10.1038/emi.2013.58.

13. Arriens C, Wren JD, Munroe ME, Mohan C. Systemic lupus erythematosus biomarkers: the challenging quest. Rheumatology (Oxford). 2017;56:i32-i45. doi: 10.1093/ rheumatology/kew407.

14. Wong CK, Ho CY, Li EK, Lam CW. Elevation of proinflammatory cytokine (IL-18, IL-17, IL-12) and Th2 cytokine (IL-4) concentrations in patients with systemic lupus erythematosus. Lupus. 2000;9:589-93. doi: 10.1191/096120300678828703.

15. Talaat RM, Mohamed SF, Bassyouni IH, Raouf AA. Th1/Th2/Th17/Treg cytokine imbalance in systemic lupus erythematosus (SLE) patients: Correlation with disease activity. Cytokine. 2015;72:146-53. doi: 10.1016/j. cyto.2014.12.027.

16. Abdel Galil SM, Ezzeldin N, El-Boshy ME. The role of serum IL-17 and IL- 6 as biomarkers of disease activity and predictors of remission in patients with lupus nephritis. Cytokine. 2015;76:280-7. doi: 10.1016/j.cyto.2015.05.007.

17. Chen DY, Chen YM, Wen MC, Hsieh TY, Hung WT, Lan JL. The potential role of Th17 cells and Th17-related cytokines in the pathogenesis of lupus nephritis. Lupus. 2012;21:138596. doi: 10.1177/0961203312457718.

18. Sun F, Teng J, Yu P, Li W, Chang J, Xu H. Involvement of TWEAK and the NF-kappaB signaling pathway in lupus nephritis. Exp Ther Med. 2018;15:2611-9. doi: 10.3892/ etm.2018.5711.

19. Schwartz N, Rubinstein T, Burkly LC, Collins CE, Blanco
I, Su L, et al. Urinary TWEAK as a biomarker of lupus nephritis: a multicenter cohort study. Arthritis Res Ther. 2009;11:R143. doi: 10.1186/ar2816.

20. Xuejing Z, Jiazhen T, Jun L, Xiangqing X, Shuguang Y, Fuyou L. Urinary TWEAK level as a marker of lupus nephritis activity in 46 cases. J Biomed Biotechnol. 2012;2012:359647. doi: 10.1155/2012/359647.

21. Reyes-Martinez F, Perez-Navarro M, Rodriguez-Matias A, Soto-Abraham V, Gutierrez-Reyes G, Medina-Avila Z, et al. Assessment of urinary TWEAK levels in Mexican patients with untreated lupus nephritis: An exploratory study. Nefrologia. 2018;38:152-60. doi: 10.1016/j. nefro.2017.04.005.

22. Raymond W, Ostli-Eilertsen G, Griffiths S, Nossent J. IL$17 \mathrm{~A}$ levels in systemic lupus erythematosus associated with inflammatory markers and lower rates of malignancy and heart damage: Evidence for a dual role. Eur J Rheumatol. 2017;4:29-35. doi: 10.5152/eurjrheum.2017.16059.

23. Wong CK, Lit LC, Tam LS, Li EK, Wong PT, Lam CW. Hyperproduction of IL-23 and IL-17 in patients with systemic lupus erythematosus: implications for Th17mediated inflammation in auto-immunity. Clin Immunol. 2008;127:385-93. doi: 10.1016/j.clim.2008.01.019.

24. Crispin JC, Tsokos GC. IL-17 in systemic lupus erythematosus. J Biomed Biotechnol. 2010;2010:943254. doi: $\quad 10.1155 / 2010 / 943254$.

25. Fragoso-Loyo H, Atisha-Fregoso Y, Nunez-Alvarez CA, Llorente L. Utility of TWEAK to assess neuropsychiatric disease activity in systemic lupus erhytematosus. Lupus. 2016;25:364-9. doi: 10.1177/0961203315610206.

Copyright $\odot 2019$ The Author(s); Published by Nickan Research Institute. This is an open-access article distributed under the terms of the Creative Commons Attribution License (http://creativecommons.org/licenses/by/4.0), which permits unrestricted use, distribution, and reproduction in any medium, provided the original work is properly cited. 